Relations industrielles

Industrial Relations

\title{
Annette DAVIES : Industrial Relations and New Technology. UWIST /Croom Helm, London, Sydney, Dover, 1986, ISBN \\ 0-7099-0882-2
}

\section{Guy Fréchet}

Volume 43, numéro 2, 1988

URI : https://id.erudit.org/iderudit/050422ar

DOI : https://doi.org/10.7202/050422ar

Aller au sommaire du numéro

Éditeur(s)

Département des relations industrielles de l'Université Laval

ISSN

0034-379X (imprimé)

1703-8138 (numérique)

Découvrir la revue

Citer ce compte rendu

Fréchet, G. (1988). Compte rendu de [Annette DAVIES : Industrial Relations and New Technology. UWIST /Croom Helm, London, Sydney, Dover, 1986, ISBN 0-7099-0882-2]. Relations industrielles / Industrial Relations, 43(2), 468-469. https://doi.org/10.7202/050422ar

Tous droits réservés @ Département des relations industrielles de l'Université Laval, 1988
Ce document est protégé par la loi sur le droit d'auteur. L’utilisation des services d'Érudit (y compris la reproduction) est assujettie à sa politique d'utilisation que vous pouvez consulter en ligne.

https://apropos.erudit.org/fr/usagers/politique-dutilisation/ 
Alienation is a very serious matter and there is much need to overcome it. The promotion of management participation and self-governmental forms at workplaces is one of the means which may be effective for socialization and education. The way how people are organized at work and how they are led, motivated, rewarded, and acknowledged has much to do with the psychological comfort. Conflicts of interest within the workplaces are actually unavoidable and the total elimination of them is neither needed nor possible.

The question is how to handle conflicts successfully using the social energy produced by them for the common benefit. In the modern societies there is more and more objective need or even necessity to reinforce the factor of cooperation at the expense of the factor of conflict. This is much dictated by the progressively complicated nature of the organizational and technical basis, as well as by the necessity to overcome waste.

The evolutionary process of a piece-meal reconstruction of society appears as much more effective than the revolutionary change, which changes radically the political and ideological superstructure, but actually is not suitable for the in-depth reconstruction of values, manners, petty interests, and semi-private affiliations. For example, the Yugoslav form of the enterprise self-government so for has not contribute much to better work habits and higher output of local workers.

Alexander J. MATEJKO

University of Alberta

Industrial Relations and New Technology, par Annette Davies, UWIST/Croom Helm, London, Sydney, Dover NH, 1986, pp., ISBN 0-7099-0882-2

Voici une recherche qui permet de lever le voile sur les impacts des changements technologiques vécus à l'intérieur d'une industrie anglaise, soit celle des brasseries. Ả première vue, le titre peut apparaître comme étant bien présomptueux pour une recherche empirique réalisée au sein d'un secteur industriel limité. Il s'explique cependant par la portée plus générale que l'auteure a bien voulu donner à sa recherche: la première moitié du livre en effet est consacrée à diverses considérations théoriques sur le débat entourant les changements technologiques, ainsi que sur le contexte des relations du travail en Grande-Bretagne. Ce n'est que dans la seconde moitié que l'auteure, pour illustrer ses considérations, analyse le cas des brasseries.

L'auteure part, comme bien d'autres, de la thèse de Braverman qu'elle critique à son tour pour la situer dans le contexte particulier des relations patronales-syndicales de la GrandeBretagne (pp. 41 et ss), surtout en ce qui touche les motivations patronales pour l'imposition d'une forme de rationalisation du travail, qui ne découlent pas entièrement d'une volonté de contrôle liée au seul «management scientifique». En revanche, les formes de rationalisation, en particulier celles qui sont permises par l'implantation de technologies de la micro-électronique, sont pour Annette Davies autant d'occasions pour les syndicats de négocier leur coopération conflictuelle avec l'entreprise. Voilà où se trouve en particulier l'intérêt principal de la recherche et son originalité, l'auteure tentant de voir si cette coopération peut être ou non l'objet d'une négociation à travers une multitude de stratégies. Parmi les stratégies en question, qui pourraient être visualisées sur un axe allant de la participation nulle à la pleine participation, toutes les méthodes traditionnelles des relations du travail se trouvent représentées. Elles sont critiquées tour à tour et l'auteure en arrive à une certaine idéalisation des méthodes qui placent la participation et un fort degré d'implication des employé(e)s au coeur même des stratégies. 
La seconde moitié est consacrée à l'analyse des résultats d'un questionnaire auprès de $\mathbf{5 0}$ des 60 brasseries du pays, complété par des entrevues effectuées dans 25 de ces établissements. Il s'agit d'une industrie qui emploie 69,000 personnes et qui est fortement syndiquée (pp. 118-120). Le schéma de la page 105 présente l'essentiel des dimensions de la recherche: la technologie et les autres variables environnementales, les comportements et conditions propres aux processus de négociation, les types de perception des relations patronales-syndicales et enfin, la satisfaction du management comme des syndicats envers le changement technologique. Le questionnaire portait sur ces divers aspects; il fut administré dans chaque cas auprès des représentants patronaux et de ceux des syndicats. Les résultats sont livrés de façon claire et les analyses statistiques sous-jacentes permettent de bien cerner les différences entre les deux points de vue (les analyses de corrélation, avec le Tau de Kendall, bien employé, permettent de voir où se situent les différences significatives).

Là où se trouve la principale faiblesse à notre avis, c'est dans le fait de mettre tous les types possibles d'application des technologies dans le même ensemble; la distinction n'est presque jamais établie entre les technologies orientées soit vers la gestion (et aux divers modes de contrôle sous-jacents), soit vers l'automatisation (et aux divers modes de production sous-jacents), sauf dans le questionnaire (question 4), qui a pu servir à construire une variable dont on ne s'est pas vraiment servi pour spécifier l'analyse. Aussi, il est difficile d'établir si les répondants parlent bien de la même chose, ceux-ci ayant pu cocher indifféremment les deux types d'application si c'était pertinent pour leur entreprise. Dans le relevé des différences entre le personnel du management et les représentants des syndicats, la confusion persiste constamment, les uns se référant davantage à la bureautique et les autres, à la production assistée par ordinateur.

En conclusion, l'auteure finit par reconnaître que même si la technologie n'est pas orientée au départ et qu'il existe des choix possibles dans la détermination de ses finalités (productivité, enrichissement des tâches, qualité de vie au travail, etc.), celles-ci restent largement liées aux prérogatives patronales. Les possibilités de réduction des heures de travail, de partage du travail ou d'allongement des vacances ne sont invariablement pas utilisées; les seuls gains partout observables furent de légères augmentations de salaires. Il s'agit d'un constat qui rejoint ce que plusieurs autres auteurs ont déjà observé ailleurs.

Davies conclut (pp. 203-205) que la perception syndicale du processus de négociation est marquée par une impression d'une forte implication au sein des processus de changement, impression qui n'a trop souvent que peu à voir avec la réalité. Elle prétend ensuite qu'un des problèmes majeurs relevés est le manque d'information permettant aux syndicats de s'impliquer dans le processus décisionnel entourant les changements technologiques. Elle déplore enfin que les politiques de main-d'oeuvre $n$ 'intègrent pas suffisamment les données des études sur les nouvelles technologies. Après avoir brossé ce tableau avec le cas des brasseries, fort intéressant en soi, le sentiment demeure que même s'il s'agit là de technologies dites «nouvelles», les enjeux et problèmes qui persistent sont tout à fait liés à ceux que l'on observe à travers les processus classiques de modernisation industrielle.

Guy FRÉCHET

IQRC

Sexual Harassment in the Workplace, by Arjun P. Aggarwal, Butterworths, Toronto, 1987, 230 pp., ISBN 0-409-80535-1

One of the most controversial and, often, complex issues in the workplace in the 1980's in Canada is sexual harassment. It knows no boundaries and its victims have suffered for long without recourse. Until recently, legislators, employers, and unions have not bothered to deal 